\title{
Low frequency, current mode programmable KHN filters using large-valued active resistors
}

\author{
Carlos Muñiz-Montero ${ }^{(1)}$, Ramón González-Carvajal ${ }^{(2)}$, Alejandro Díaz-Sánchez ${ }^{(1)}$ and J. Miguel Rocha ${ }^{(1)}$ \\ (1) Dpto. de Electrónica. \\ Instituto Nacional de Astrofísica Optica y Electrónica \\ Puebla, MEXICO \\ cmuniz, adiazsan,jmr@inaoep.mx \\ (2) Dpto. de Ingeniería Electrónica \\ Escuela Superior de Ingenieros, Universidad de Sevilla \\ Sevilla, SPAIN \\ carvajal@gte.esi.us.es
}

\begin{abstract}
The present work proposes a new current-mode, programmable Kerwin Huelsman Newcomb (KHN) filter suitable for very low frequency processing. The proposed implementation, developed with only six current mirrors and two large-valued, programmable active resistors, solves some of the more challenging problems of low frequency filtering. HSPICE simulation results using BSIM3.3 models for a $0.5-\mu \mathrm{m}$ CMOS technology, are shown. Center frequencies in the range of $12.4 \mathrm{~Hz}-33 \mathrm{~Hz}$, total harmonic distortion less than $1 \%$, and static power consumption of $1.05 \mathrm{~mW}$, were observed.
\end{abstract}

\section{INTRODUCTION}

In low frequency applications, such as analog processing of biomedical signals, analog filters are necessary to reject unwanted signals, among them, large DC offsets generated by sensors and flicker or out band noise. Also, the bandwidth of most of the biomedical signals is in the range of $0.1 \mathrm{~Hz}-$ $10 \mathrm{kHz}$ and, due to the typical requirements of biomedical applications, programmability is mandatory [1]. Unfortunately, the design of programmable integrated filters with bandwidths below $1 \mathrm{kHz}$ is not trivial, especially if other design specifications such as low voltage implementation, low noise, high dynamic range, low distortion, small area and reduced power consumption, must be satisfied. The main challenge comes from the lack of large time constants available in integrated circuits. To overcome this lack, there have been used impractical techniques such as: use of external capacitors (additional output pads), capacitance multiplication (coarse programmability that is only possible with discrete control), subthreshold operation of MOS transistors (prone to present big noise contributions and temperature dependency), and techniques for current division and current cancellation (prone to present mismatch and large offset components)

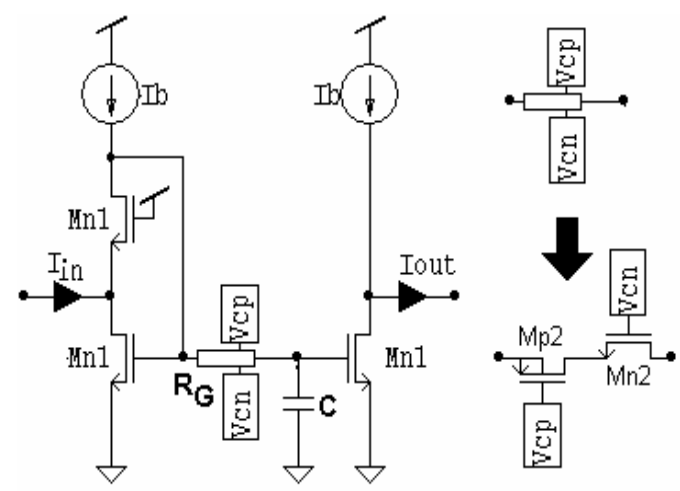

(a)

(b)

Figure 1. (a) Low frequency, low pass filter (LPF). (b) Large-valued, programmable active resistor realization.

[2-3]. Therefore, the use of very large-valued active resistors, especially if their values can be easily programmed, looks like a more pragmatic alternative. Fortunately, the implementation of integrated large-valued active resistors is possible and has been recently employed in new offset compensation techniques [4] and polarization strategies [5]. This paper proposes the use of large-valued active resistors to obtain a new low-frequency, current-mode programmable KHN filter.

\section{LOW-PASS Filter For LOW FREQUENCY OPERATION}

Let's consider the low-pass filter in Fig. 1a which is based on the flipped-voltage follower structure [6]. If $R_{G}$ and $C$ are chosen much bigger than $r_{d s, M N 1}$ and all the parasitic capacitances, respectively, then we get:

Work supported by CONACyT, Mexico, under Grant I-37470-A. 


$$
A(s)=\frac{I_{\text {out }}}{I_{\text {in }}} \approx-\frac{1 / R_{G} C}{s+1 / R_{G} C}=-\frac{\psi}{s+\psi}
$$

It can be noticed, under these assumptions, that expression (1) does not depend of the polarization current. Also, the transmission zero associated to $C_{g d, M n I}$ is located at frequencies far above of the main pole. Therefore, the described low pass filter is a good approximation of a quasiideal integrator. The implementation of $R_{G}$ is illustrated in Fig. 1b [5]. Transistors $M_{n 2}$ and $M_{p 2}$ are biased in subthreshold operation by voltages $V_{c n}$ and $V_{c p}$. The effective resistance always remains on the order of gigaohms, even in the case of large voltage variations at their terminals. Nevertheless, this situation never happens due to the current to voltage compression realized by transistors $M_{n l}$ operating in saturation. Therefore, the distortion associated to the nonlinear behavior of $R_{G}$ is minimized. Since $R_{G}$ is very large, a small capacitor can be used to obtain a cutoff frequency in the order of tenths of hertz.

The subthreshold operation of $M_{n 2}$ and $M_{p 1}$ forces $R_{G}<<R_{B}$ (where $R_{B}$ is the diffusion resistance), in order to avoid a parasitic resistive divider. Unfortunately, this parasitic load effect produces a small voltage drop in $R_{G}$ [7], and consequently a small voltage difference between the gates of both $M_{n 1}$ transistors. This way, if the gain of the current mirror is large, a considerable DC systematic offset can appear at the output current. A solution for this problem will be described in the next section.

\section{LOW FreQuency, CURRENT-Mode KHN FILTER}

The KHN filter has extreme flexibility, good performance and low sensitivities [8]. A block diagram of an approximation of this filter, using the low pass filter presented in the previous section, is illustrated in Fig. 2. With a straightforward analysis of this diagram (without considering the systematic offset compensation array), we obtain:

$$
\begin{gathered}
A_{H P}(s)=\frac{I_{H P}}{I_{\text {in }}}=\frac{H_{H P}(s+\psi)^{2}}{s^{2}+\psi\left(2+a_{1} H\right) s+\psi^{2}\left(1+a_{1} H+a_{0} H^{2}\right)} \\
A_{B P}(s)=\frac{I_{B P}}{I_{\text {in }}}=\frac{-H_{B P} H \psi(s+\psi)}{s^{2}+\psi\left(2+a_{1} H\right) s+\psi^{2}\left(1+a_{1} H+a_{0} H^{2}\right)} \\
A_{L P}(s)=\frac{I_{L P}}{I_{\text {in }}}=\frac{H_{L P} H^{2} \psi^{2}}{s^{2}+\psi\left(2+a_{1} H\right) s+\psi^{2}\left(1+a_{1} H+a_{0} H^{2}\right)}
\end{gathered}
$$

where the subindexes HP, BP and LP correspond to highpass, band-pass and low-pass signals, respectively. The natural frequency and quality factor are:

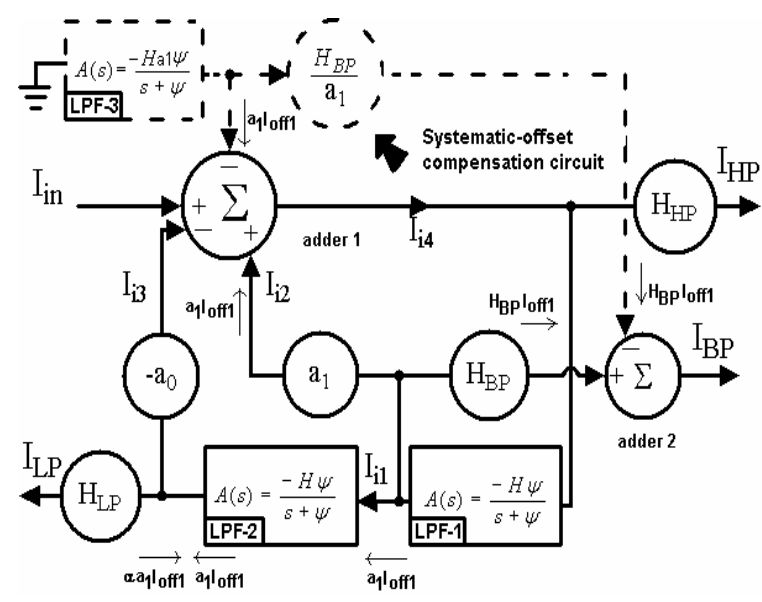

Figure 2. Blocks diagram of the low frequency KHN filter.

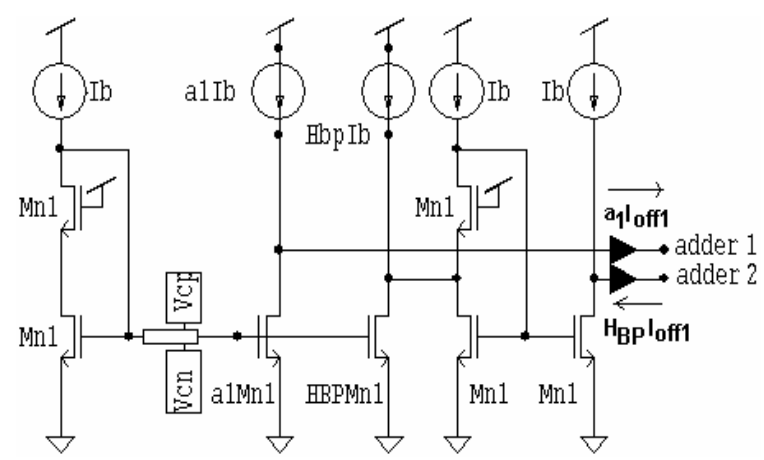

Figure 3. Systematic-offset, compensation circuit

$$
\begin{array}{r}
\omega_{n}=\psi \sqrt{1+a_{1} H+a_{0} H^{2}} \\
Q=\frac{\sqrt{1+a_{1} H+a_{0} H^{2}}}{2+a_{1} H}
\end{array}
$$

From (5) is observed that the natural frequency can be programmed modifying the parameter $\psi=1 /\left(R_{G} C\right)$ through the voltages $V_{c n}$ and $V_{c p}$.

The systematic offset compensation array illustrated in Fig. 2 and implemented in Fig. 3 for $H=1$ works as follows. The systematic offset generated by the block LPF-1, $I_{\text {off }}$, is amplified by factors $a_{l}$ or $H_{B P}$ during the generation of the signals $I_{i 2}$ and $I_{B P}$, respectively. Consequently, it is necessary subtract DC components $a_{1} I_{\text {off } 1}$ and $H_{B P} I_{\text {off }}$ to $I_{i 2}$ and $I_{B P}$ to compensate the systematic offset components in $I_{H P}$ and $I_{B P}$. These components are generated by the blocks LPF-3 (because it is identical to LPF-1, but with a gain $a_{1}$ ) and $\mathrm{H}_{\mathrm{BP}} / \mathrm{a}_{1}$ (with overall gain $\mathrm{H}_{\mathrm{BP}}$ ), and then are subtracted of $I_{i 2}$ and $I_{B P}$ through the inverting inputs of the blocks adder 1 and adder 2. Note that LPF-3 does not need a capacitor because it is in a DC trajectory. 


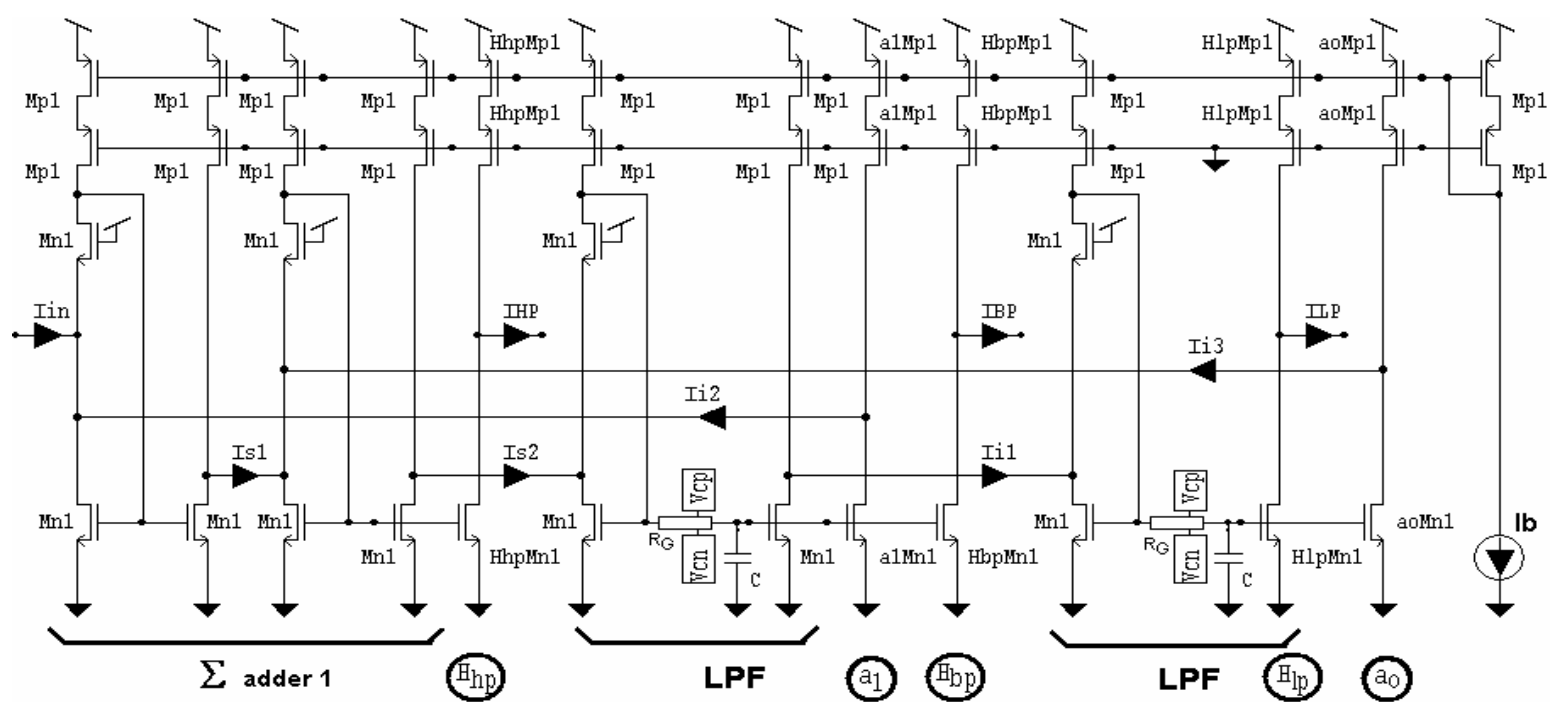

Figure 4. Implementation with $H=1$ of the current-mode, low frequency KHN filter of figure $2 . H_{h p}, H_{b p}, H_{l p}, a_{l}$ and $a_{0}$ are the number of transistors connected in parallel.

Once compensated the systematic offset in $I_{H P}$ and $I_{B P}$, the systematic offset in $I_{L P}$ is cancelled because the offset component generated by LPF-1, $I_{\text {offl }}$, is inverted by LPF-2 (with a small error $\varepsilon=1-\alpha$, where $\alpha \approx 1$, caused by the small voltage drop in $R_{G}$; the error is small because LPF-2 has unity gain) and then added to the offset generated by LPF-2, which is $I_{\text {off } 1}$ too. Therefore, the systematic offset in $\mathrm{I}_{\mathrm{LP}}$ results in:

$$
\left.I_{L P}\right|_{o f f s e t}=H_{L P}(1-\alpha) I_{o f f 1}
$$

Figure 4 shows an implementation of the KHN filter with $H=1$ that uses four current mirrors, two of them implemented as integrators. Two additional mirrors are used to incorporate the offset compensation array of Fig. 3.

\section{RESUlts}

The low pass filter of figure 1 and the KHN filter of figures 3 and 4 have been designed using BSIM3.3 models for a 0.5 $\mu \mathrm{m}$ CMOS AMI process through MOSIS $\left(\mathrm{V}_{\mathrm{THn}}=0.65 \mathrm{~V}\right.$, $\left.\left|\mathrm{V}_{\text {THp }}\right|=0.95 \mathrm{~V}\right)$. The design details are illustrated in Table I. The voltage drop of $R_{G}$ and the corresponding systematic offset current of the low pass filter, for a sweep of $V_{c p}$ between $0.1 \mathrm{~V}$ and $0.3 \mathrm{~V}$, are shown in Fig. 5. Maximum errors of $15 \mathrm{mV}$ and $2 \mu \mathrm{A}$ were observed when $\mathrm{V}_{\mathrm{cp}}$ is equal to $0.3 \mathrm{~V}$, where $\mathrm{M}_{\mathrm{p} 1}$ is nearer of the cutoff operation and the relation $R_{G}<<R_{B}$ is not satisfied. The frequency responses for the LP, BP and HP signals are illustrated in Fig. 6. Cutoff and center frequencies in the range $12.4 \mathrm{~Hz}-33 \mathrm{~Hz}$ were obtained with a sweep of $\mathrm{V}_{\mathrm{cp}}$ between $0.1 \mathrm{~V}$ and $0.3 \mathrm{Vin}$ increments of $20 \mathrm{mV}$.
TABLE I. DESIGN DETAILS

\begin{tabular}{|c|c|}
\hline $\mathrm{M}_{\mathrm{n} 1}, \mathrm{M}_{\mathrm{n} 2}, \mathrm{M}_{\mathrm{p} 1}, \mathrm{M}_{\mathrm{p} 2}$ & $\begin{array}{c}\mathrm{W} / \mathrm{L}(\mu \mathrm{m} / \mu \mathrm{m})= \\
9 / 1.2,12 / 3,18 / 1.2,12 / 3\end{array}$ \\
\hline $\mathrm{a}_{0}, \mathrm{a}_{1}, \mathrm{H}, \mathrm{C}$ & $10,1,1,10 \mathrm{pF}$ \\
\hline $\mathrm{H}_{\mathrm{HP}}, \mathrm{H}_{\mathrm{BP}}, \mathrm{H}_{\mathrm{LP}}$ & $12,12,12$ \\
\hline $\mathrm{I}_{\mathrm{b}}, \mathrm{V}_{\mathrm{DD}}, \mathrm{V}_{\mathrm{cn}}, \mathrm{V}_{\mathrm{cp}}$, & $10 \mu \mathrm{A}, 1.5 \mathrm{~V}, 1.5 \mathrm{~V}$, \\
$\mathrm{V}_{\mathrm{cp}} \in(0.1 \mathrm{~V}, 0.3 \mathrm{~V})$
\end{tabular}

For input signals of amplitude from $1 \mu \mathrm{A}$ to $7 \mu \mathrm{A}$, and frequencies of $5 \mathrm{~Hz}, 10 \mathrm{~Hz}$ and $40 \mathrm{~Hz}$, for the $\mathrm{LP}, \mathrm{BP}$ and HP filters, respectively, the total harmonic distortions are shown in Fig. 7. The LP and HP signals present a THD below $1.2 \%$, while the BP signal presents a THD in the worst case of $5.5 \%$. The overall static power consumption was of $1.05 \mathrm{~mW}$. In Fig. 8 the systematic offset currents can be observed. In the worst case, the offset was below $160 \mathrm{nA}$. In an uncompensated version of the circuit of Fig. 4, offsets of until $6.2 \mu \mathrm{A}$ were observed in $\mathrm{I}_{\mathrm{BP}}$. Therefore, systematic offset compensations of until $99.5 \%$ were obtained, as is illustrated in Fig. 9.

\section{CONCLUSIONS}

A new strategy to design low-frequency, KHN programmable integrated filters has been presented. This technique uses large-valued active resistors to overcome the principal challenges of the other strategies in the literature, allowing a compact and easy circuit realization, with low hardware complexity, low power consumption and fully integrated capacitors. An efficient and compact structure to compensate the systematic offset contributions has been presented too. 

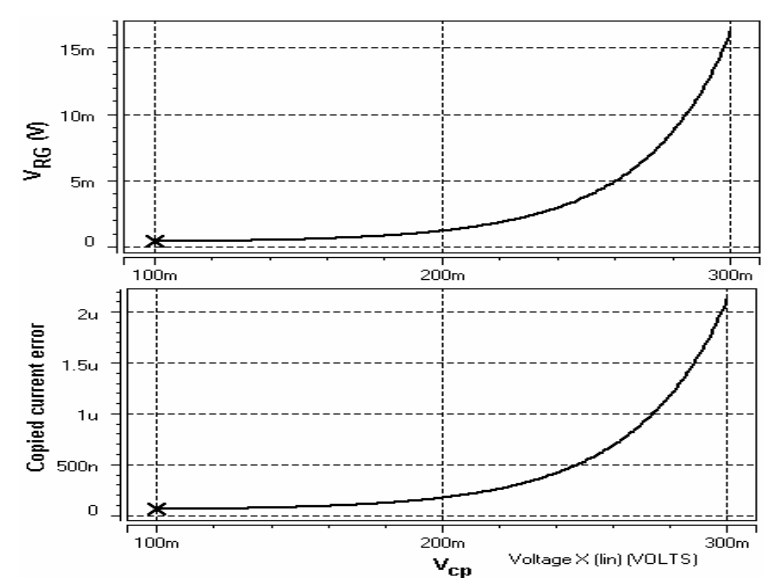

Figure 5. Voltage drop in RG and the corresponding offset current.

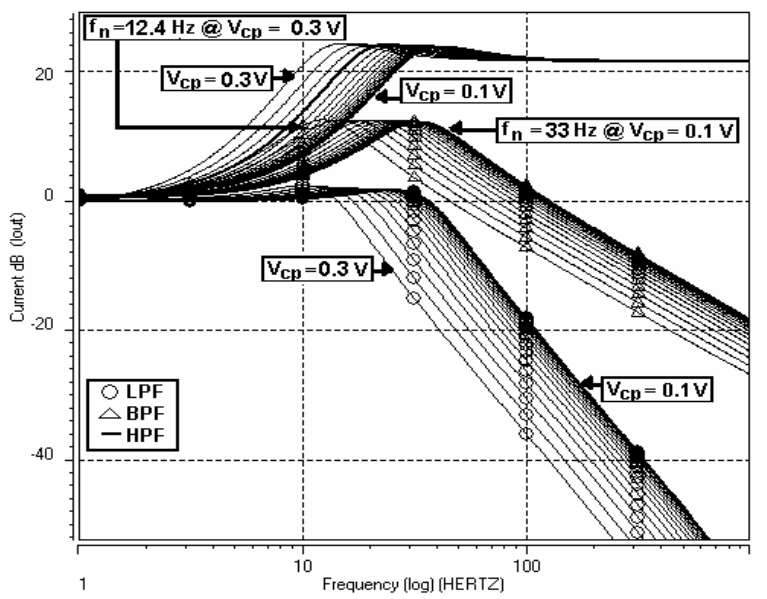

Figure 6. LP, BP and HP responses of the KHN filter with a sweep of Vep from $0.1 \mathrm{~V}$ to $0.3 \mathrm{~V}$ in increases of $20 \mathrm{mV}$.

\section{REFERENCES}

[1] J. D. Bronzino, The Biomedical Engineering Handbook, CRC Press Inc. and IEEE Press, 1995.

[2] S. Solis-Bustos, J. Silva-Martínez, F. Malloberti and E. SánchezSinencio, "A 60 dB Dynamic Range Sixth Order $2.4 \mathrm{~Hz}$ Lowpass Filter for Medical Applications," IEEE Transactions on Circuits and Systems Part II, Vol. 47, No. 12, pp. 1391-1398, December 2000.

[3] J. Silva-Martínez and S. Solis-Bustos, "Designs considerations for high performance very low frequency filters," Proc. of the 50th International Symposium on Circuits and Systems, ISCAS 99, Vol II, pp. 648-651, Orlando, USA, June 1999.

[4] C. Muñiz Montero, R. González Carvajal and A. Díaz Sánchez, "Offset Compensation Using Unbalanced Polarization," Proc. of the 57th International Symposium on Circuits and Systems, ISCAS 2006, pp. 1868-1871, Island of Kos, Grece, May 2006.

[5] M. Bikumandla, J. Ramírez-Angulo, C. Urquidi, R. G. Carvajal and A. J. Lopez-Martin. "Biasing CMOS amplifiers using MOS transistors in subthreshold region," in IEICE Electronics Express, vol. 1 no. 12, September 25, 2004.

[6] R.G Carvajal, et. al., "The flipped voltage follower: a useful cell for low-voltage low-power circuit design," in IEEE Transactions on Circuits and Systems I: Regular Papers, vol. 52, no. 7, pp.1276 1291, July 2005.

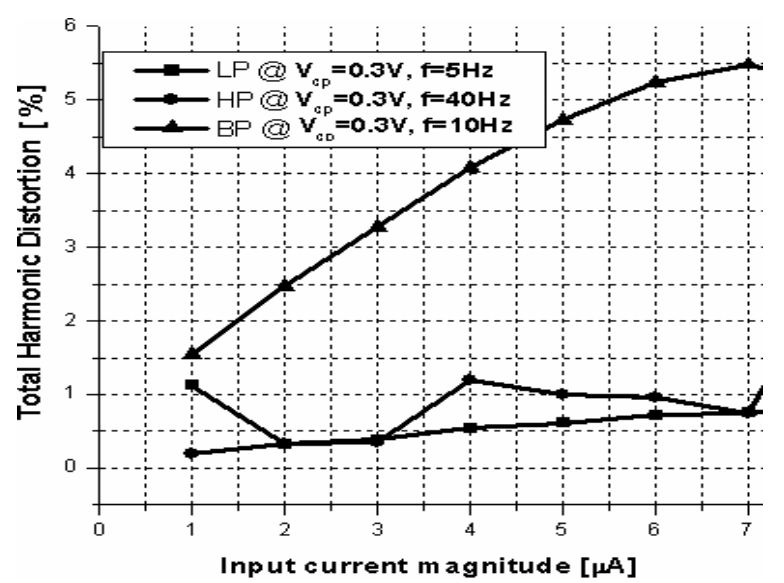

Figure 7. Total harmonic distortion of the LP, BP and HP outputs.

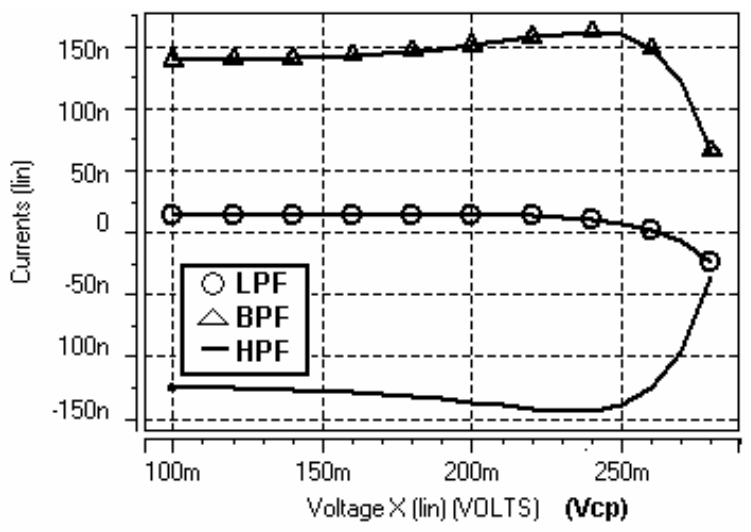

Figure 8. Systematic offset in the LP, BP and HP outputs.

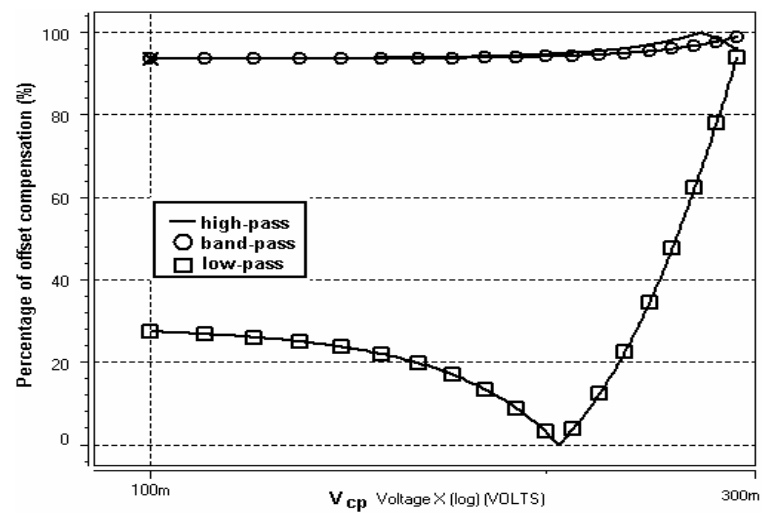

Figure 9. Systematic offset compensation

[7] I. Seo and R. M Fox, "Comparison of Quasi-/Pseudo-Floating Gate Techniques and Low-Voltage Applications," in Analog Integrated Circuits and Signal Processing, V. 47, pp. 183-192. 2006.

[8] L.P. Huelsman and P.E. Allen, Introduction to the theory and design of active filters, McGraw-Hill Book Co. USA, 1980. 